\title{
Author Correction: Clonal architecture in mesothelioma is prognostic and shapes the tumour microenvironment
}

Min Zhang, Jin-Li Luo, Qianqian Sun, James Harber, Alan G. Dawson (1), Apostolos Nakas, Sara Busacca, Annabel J. Sharkey (D), David Waller, Michael T. Sheaff, Cathy Richards, Peter Wells-Jordan, Aarti Gaba, Charlotte Poile, Essa Y. Baitei, Aleksandra Bzura, Joanna Dzialo (D), Maymun Jama, John Le Quesne, Amrita Bajaj, Luke Martinson, Jacqui A. Shaw (1), Catrin Pritchard, Tamihiro Kamata (B), Nathaniel Kuse, Lee Brannan, Pan De Philip Zhang, Hongji Yang (1), Gareth Griffiths, Gareth Wilson, Charles Swanton (D), Frank Dudbridge (D), Edward J. Hollox (1) \& Dean A. Fennell (1)

Correction to: Nature Communications https://doi.org/10.1038/s41467-021-21798-w, published online 19 March 2021.

The original version of this Article contained an error in the spelling of the author Luke Martinson, which was incorrectly given as Luke Martison.

This has now been corrected in both the PDF and HTML versions of the Article.

Published online: 07 June 2021

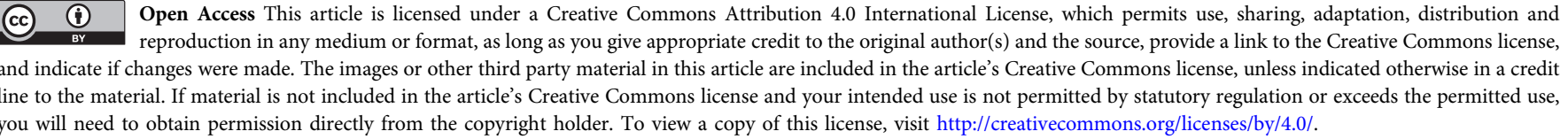
you will need to obtain permission directly from the copyright holder. To view a copy of this license, visit http://creativecommons.org/licenses/by/4.0/.

(C) The Author(s) 2021 